\title{
STEADY MHD MIXED CONVECTION NEWTONIAN FLUID FLOW ALONG A VERTICAL STRETCHING CYLINDER EMBEDDED IN POROUS MEDIUM
}

\author{
Sharad Sinha ${ }^{1} \square$ (D) and R. S. Yadav ${ }^{2}$ \\ ${ }^{1}$ Department of Mathematics, University of Rajasthan, Jaipur, India \\ ${ }^{2}$ Department of Mathematics, University of Rajasthan, Jaipur, India
}
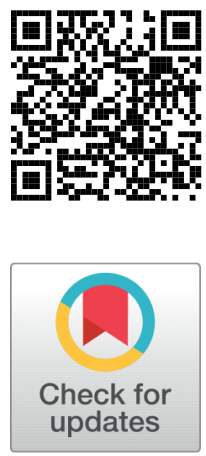

Received 25 June 2021

Accepted 10 July 2021

Published 21 July 2021

Corresponding Author

Sharad Sinha, sharadsinha89@gm ail.com

DOI $10.29121 /$

ijetmr.v8.i7.2021.990

Funding: This research received no specific grant from any funding agency in the public, commercial, or not-for-profit sectors.

Copyright: (C) 2021 The Author(s). This is an open access article distributed under the terms of the Creative Commons Attribution License, which permits unrestricted use, distribution, and reproduction in any medium, provided the original author and source are credited.

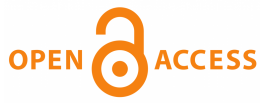

\section{ABSTRACT}

A viscous electrically conducting fluid is considered and its steady mixed convective flow along a vertical stretching cylinder is investigated. It is assumed that the cylinder is embedded in a porous medium and, external magnetic field, heat source/sink are also taken into account. Suitable similarity transformations are used to reduce the governing equations and associated boundary conditions into a system of nonlinear ordinary differential equations. This system along with the boundary conditions is solved by fourth order RungeKutta method with shooting technique. Variations in fluid velocity and temperature due to various physical parameters such as heat source/sink parameter, mixed convection parameter, magnetic parameter are presented through graphs. Effect of these parameters on dimensionless shear stress and rate of heat transfer are discussed numerically through tables.

Keywords: Stretching Cylinder, Newtonian Fluid, Heat Source, Magnetic Field

\section{INTRODUCTION}

MHD mixed convective flow along a stretching vertical cylinder has many applications in areas such as heat exchangers, geothermal power generation, nuclear reactors, drilling operations, insulation system and plastic products formation, polymer processing units, etc.

Mixed convection with heat source/sink along a vertical stretching cylinder has been investigated by a few researchers compared to the horizontal stretching cylinder. Crane Crane (1970) was the first who considered flow past a stretching plate. Chen and Mucoglu Chen and Mucoglu (1975) investigated buoyancy effects on forced convection along a vertical cylinder. Cheng Cheng (1977) took a horizontal flat plate in a saturated porous medium and analyzed the mixed convective flow along it. Wang Wang (1984) extended the study of Crane Crane (1970) and obtained an exact 
similarity solution for three-dimensional fluid flow caused by the stretching boundary. Grubka and Bobba Grubka and Bobba (1985) considered a linearly stretching continuous surface and discussed the heat transfer with power law temperature distribution. Vajravelu Vajravelu (1994) numerically analyzed the convective flow and heat transfer of viscous fluid along a vertical stretching surface with suction or blowing. Ganesan and Rani Ganesan et al. (2000) studied the applied magnetic field effects on unsteady mixed convective flow past a vertical cylinder. Steady linear and nonlinear convection in a micropolar fluid was discussed by Siddheshwar and Srikrishna Siddheshwar et al. (2003). Chang Chang (2008) considered micropolar fluid flow along a vertical slender cylinder and investigated free convective heat transfer. Ishak and Nazar Ishak et al. (2009) discussed axisymmetric boundary layer flow of a viscous incompressible fluid along a continuously stretching cylinder. Aydin and Kaya Aydin and Kaya (2011) analyzed the heat transfer characteristic in steady laminar mixed convective flow past a vertical slender cylinder. Wang Wang (2012) extended his own study Wang (1984) by considering a vertical stretching cylinder. He discussed natural convection and obtained both analytic and numerical solutions of the problem. Patil et al. Patil et al. (2012) considered a permeable nonlinear stretching vertical slender cylinder and inspected the unsteady mixed convective flow with buoyancy force and thermal diffusion. Proceeding in the same order Mishra and Singh Mishra et al. (2014) used momentum and thermal slip and investigated the flow and heat transfer over a permeable shrinking cylinder. Simultaneously, effects of heat source and porous medium on MHD free convective flow over a horizontal stretching cylinder were analyzed by Yadav and Sharma Yadav et al. (2014). Hayat et al. Hayat et al. (2015) studied mixed convective flow of viscoelastic fluid due to a vertical stretching cylinder. In their study they considered temperature dependent thermal conductivity. MHD boundary layer slip flow over a permeable stretching cylinder was explained by Reddy and Das Reddy and Das (2016). In their research they also included chemical reaction and investigated by artificial neural network. Following this Sohut et al. Sohut et al. (2017) investigated the chemical reaction and heat source effects on boundary layer over a vertical stretching cylinder. In our previous paper Sharma et al. Sharma et al. (2018), we have investigated heat generation/absorption on MHD mixed convective stagnation point flow in the presence of external magnetic field. Afterwards Vinita and Poply Vinita (2019) discussed steady MHD nanofluid slip flow past a stretching cylinder with heat generation. They explained thermophoresis and Brownian motion effects on the flow and heat, mass transfer.

Motivated by the above works here we have taken a viscous incompressible electrically conducting Newtonian fluid and attempting to investigate the steady mixed convection flow over a vertical stretching cylinder. Moreover, it is assumed that the cylinder is embedded in porous medium in the presence of magnetic field and heat source/sink. For the validation of the results of the present study, these are compared with the earlier published results and found to be in good agreement. 


\section{MATHEMATICAL FORMULATION}

Consider steady laminar boundary layer flow of a viscous incompressible electrically conducting Newtonian fluid past a stretching vertical cylinder. Here it is assumed that the radius of the cylinder is $u_{w}(x)=U x / l$,

where $l$ where is the characteristic length and $U$ is the reference velocity. The coordinate system is taken such that $r$-axis is measured in the radial direction and axis of the cylinder represents the -axis. An external magnetic field $B$ is applied along the $r$-axis. The induced magnetic field is neglected as the magnetic Reynolds number is considered very small. In view of the above assumptions, the governing equations of conservation of mass, momentum and energy are given as

$$
\begin{aligned}
& \frac{\partial}{\partial x}(r u)+\frac{\partial}{\partial r}(r v)=0, \\
& u \frac{\partial u}{\partial x}+v \frac{\partial u}{\partial r}=\frac{v}{r} \frac{\partial}{\partial r}\left(r \frac{\partial u}{\partial r}\right)-\frac{\sigma B^{2}}{\rho} u-\frac{v}{K_{p}} u+g \beta\left(\mathrm{T}-\mathrm{T}_{\infty}\right), \\
& u \frac{\partial T}{\partial x}+v \frac{\partial T}{\partial r}=\frac{\alpha}{r} \frac{\partial}{\partial r}\left(r \frac{\partial T}{\partial r}\right)+\frac{Q}{\rho C_{p}}\left(T-T_{\infty}\right),
\end{aligned}
$$

where the velocity components along $x$ and $r$-axis are $u$ and $v$, respectively. $\rho$ is density of the fluid, $\sigma$ is electrical conductivity, $\beta$ is the coefficient of thermal expansion, $g$ is acceleration due to gravity, $\nu$ is the kinematic viscosity, $K_{p}$ is the permeability of the porous medium, $C_{p}$ is the specific heat at constant pressure, $Q$ is the volumetric rate of heat source/sink, $\alpha$ is the thermal diffusivity, $T$ and $T_{\infty}$ are temperature within boundary layer and in free stream, respectively.

The associated boundary conditions are

$$
\left.\begin{array}{l}
r=a: u=u_{w}(x)=U \frac{x}{l}, v=0, \quad T=T_{w}(x)=T_{\infty}+b\left(\frac{x}{l}\right), \\
r \rightarrow \infty: \quad u \rightarrow 0, \quad T \rightarrow T_{\infty},
\end{array}\right\}
$$

where $b$ is a constant. To solve the governing equations along with boundary conditions we have used the following parameters and similarity variables:

$$
\left.\begin{array}{l}
\eta=\frac{r^{2}-a^{2}}{2 a}\left(\frac{u_{w}}{v x}\right)^{1 / 2}, \quad \psi=\left(v x u_{w}\right)^{1 / 2} a f(\eta), \quad \theta(\eta)=\frac{T-T_{\infty}}{T_{w}-T_{\infty}}, \\
u=\frac{1}{r} \frac{\partial \psi}{\partial r}, \quad v=-\frac{1}{r} \frac{\partial \psi}{\partial x},
\end{array}\right\}
$$


where $\eta$ is the similarity variable and $\psi$ is the stream function? Using equation (5) into momentum and energy equations (2) and (3) respectively, we get

$$
\begin{aligned}
& (1+2 \delta \eta) f^{\prime \prime \prime}+f f^{\prime \prime}+2 \delta f^{\prime \prime}-\left(f^{\prime}\right)^{2}-(M+K) f^{\prime}+\lambda \theta=0, \\
& (1+2 \delta \eta) \theta^{\prime \prime}+2 \delta \theta^{\prime}+\operatorname{Pr}\left(f \theta^{\prime}-f^{\prime} \theta\right)+\operatorname{Pr} S \theta=0,
\end{aligned}
$$

where the prime is differentiation with respect to $\eta, \lambda\left\{=\frac{g \beta b \iota}{v^{2}}\right\}$ is the mixed convection parameter, $K\left\{=\frac{\nu \iota}{U K_{p}}\right\}$ is the permeability parameter, $M\left\{=\frac{\sigma B^{2} \iota}{\rho^{U}}\right\}$ is the magnetic parameter, $\delta\left\{=\left\{\frac{\nu \iota}{\alpha^{2} U}\right\}^{1 / 2}\right\}$ is the curvature parameter, $\operatorname{Pr}\left\{=\frac{\nu}{\alpha}\right\}$ is the Prandtl number and $S\left\{=\frac{Q \iota}{U \rho C_{p}}\right\}$ is the heat generation/absorption parameter.

Here, continuity equation (1) is identically satisfied. Using equation (5), the boundary conditions (4) are reduced to

$f(0)=0, f^{\prime}(0)=1, \theta(0)=1, f^{\prime}(\infty) \rightarrow 0, \theta(\infty) \rightarrow 0$.

Equations (6) and (7) are nonlinear coupled differential equations with boundary conditions (8). Here we have used fourth order Runge-Kutta method with shooting technique to solve these equations.

\section{NUMERICAL SOLUTION}

According to Runge-Kutta method equations (6) and (7) with boundary conditions (8) are decomposed into system of equations of order one. By denoting $f, f^{\prime}, f^{\prime \prime}, \theta$ and $\theta^{\prime}$ by $f_{1}, f_{2}, f_{3}, f_{4}$ and $f_{5}$ the following system of equations is obtained:

$$
\begin{aligned}
& f_{1}^{\prime}=f_{2}, \\
& f_{2}^{\prime}=f_{3}, \\
& f_{3}^{\prime}=\frac{1}{(1+2 \delta \eta)}\left[f_{2}^{2}+(M+K) f_{2}-f_{1} f_{3}-2 \delta f_{3}-\lambda f_{4}\right],
\end{aligned}
$$




$$
\begin{aligned}
& f_{4}^{\prime}=f_{5}, \\
& f_{5}^{\prime}=\frac{1}{(1+2 \delta \eta)}\left[\operatorname{Pr}\left(f_{2} f_{4}-f_{1} f_{5}-S f_{4}\right)-2 \delta f_{5}\right],
\end{aligned}
$$

and associated boundary conditions are

$$
f_{1}(0)=0, f_{2}(0)=1, f_{4}(0)=1, f_{2}(\infty) \rightarrow 0, f_{4}(\infty) \rightarrow 0
$$

In process to solve the system numerically, we require five conditions at $\eta=0$, but here only three conditions are available. Apart from these three conditions two conditions are given at $\eta \rightarrow \infty$. In pursuance of shooting technique, we assume that values of $f_{3}$ and $f_{5}$ at $\eta=0$ are $s_{1}$ and $s_{2}$. To check the authenticity of these assumed values, we compute $f_{2}$ and $f_{4}$ at $\eta \rightarrow \infty$ with the help of given and assumed initial conditions and match it with the values given in (14). The process is repeated until the result of the desired accuracy is obtained.

In the study physical quantities of interest are dimensionless shear stress and rate of heat transfer at the wall. Expression for these is given below

\section{Skin friction Coefficient}

The shearing stress at the surface is given by

$$
\tau_{w}=\mu\left(\frac{\partial u}{\partial r}\right)_{r=a}
$$

where $\mu$ is the coefficient of viscosity?

The skin friction coefficient at the surface is given by

$$
\begin{aligned}
& C_{f}=\frac{2 \tau_{w}}{\rho u_{w}^{2}} \\
& \Rightarrow \frac{1}{2} C_{f} \operatorname{Re}^{1 / 2}=f^{\prime \prime}(0),
\end{aligned}
$$


where $\operatorname{Re}\left\{=\frac{l U}{\nu}\right\}$ is the Reynolds number?

\section{Heat transfer Coefficient}

The heat flux at the surface is given by

$$
q_{w}=-\kappa\left(\frac{\partial T}{\partial r}\right)_{r=a},
$$

where $K$ is thermal conductivity of the fluid?

The Nusselt number is defined as

$$
\begin{aligned}
& N u_{x}=\frac{x}{\kappa} \frac{q_{w}}{\left(T_{w}-T_{\infty}\right)} \\
& \Rightarrow \frac{N u_{x}}{\operatorname{Re}^{1 / 2}}=-\theta^{\prime}(0) .
\end{aligned}
$$

Variation in fluid velocity and temperature due to various physical parameters are presented through figures. The dimensionless shear stress and rate of heat transfer at the surface are discussed numerically through table for various values of the physical parameters.

\section{RESULTS AND DISCUSSIONS}

Figure 1 illustrates the effect of magnetic parameter on fluid velocity. It is seen from the figure that fluid velocity decreases with increasing values of magnetic parameter. The reason behind this is larger magnetic parameter corresponds to stronger Lorentz force which resist the motion of the fluid. This decreasing velocity causes lesser skin friction as noticed from Table 2 . Effect of curvature parameter on fluid velocity is depicted in Figure 2 . The curvature parameter is inversely proportional

\begin{tabular}{|c|c|c|}
\hline$P r$ & Ishak and Nazar Ishak et al. (2009) & Present Study \\
\hline 1 & 1.0000 & 1.00000 \\
\hline 10 & 3.7207 & 3.719976 \\
\hline
\end{tabular}
to the radius of the cylinder, so for higher curvature parameter there will be lesser interaction between the fluid particles and the surface, as a result velocity goes up.

It is observed from Figure 3 that fluid temperature increases with larger magnetic parameter. As explain earlier that Lorentz force works as a resistance and retards the 
fluid motion, due to this resistance some amount of energy transforms into heat and increases the temperature. This increasing temperature reduces the temperature difference between the surface and the fluid, and because of this Nusselt number decrease, as noted from Table 2 . Figure 4 illustrates that increasing permeability parameter causes a drop in temperature of the fluid. Larger permeability parameter shows more assistance to the fluid to flow through the porous medium, due to this orderly mannered flow, temperature goes down. The effect of curvature parameter on fluid temperature is shown through Figure 5 . It is quoted in the explanation of Figure 2 that fluid velocity increases with increasing curvature parameter. Due to this increased velocity there will be more kinetic energy and, the temperature is defined by the average kinetic energy therefore temperature increases. Impact of Prandtl number on fluid temperature is examined in Figure 6 . For larger values of Prandtl number, kinematic viscosity dominates the thermal diffusivity and this lesser thermal diffusivity results in temperature drop near the surface. This decreasing temperature creates more temperature difference between the surface and the fluid and as an outcome Nusselt number increase. It is noted from Figure 7 that fluid temperature increases with increasing heat generation parameter. This increasing temperature corresponds to low temperature difference and Nusselt number. Figure 8 is plotted to see the impact of mixed convection parameter on fluid temperature. As the mixed convection parameter increases, Grashof number dominates the Reynolds number and, in outcome more buoyancy force works on flowing fluid. This increased buoyancy force gives a hike to the temperature near the surface.

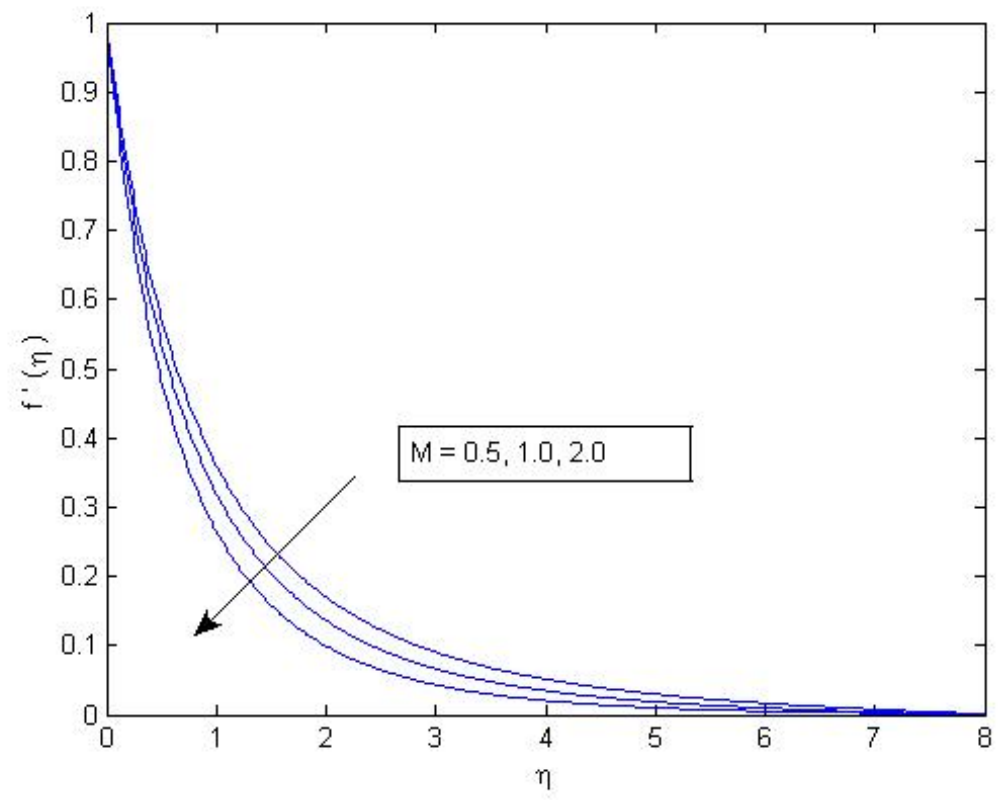

Figure 1 Velocity distribution versus $\eta$ when $\operatorname{Pr}=7, S=0.5, \lambda=0.2, \beta=0.5, \delta=0.5$. 


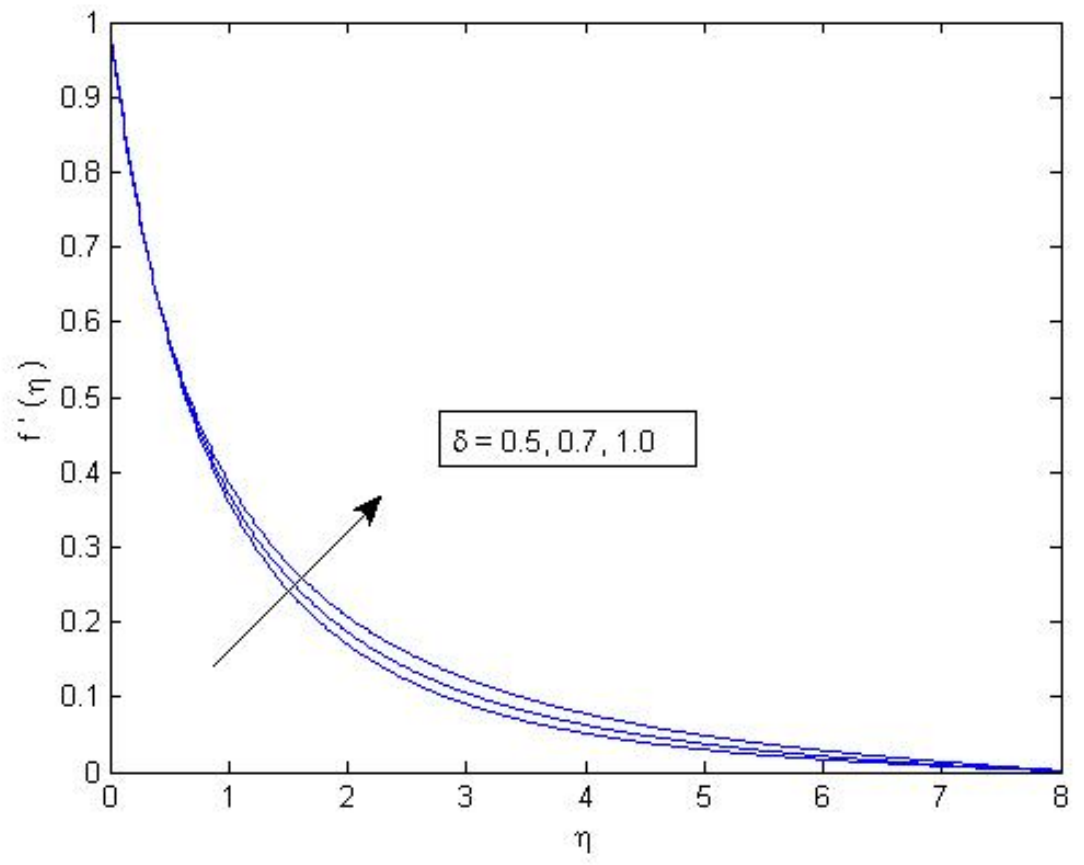

Figure 2 Velocity distribution versus $\eta$ when $\operatorname{Pr}=7, S=0.5, M=0.5, \lambda=0.2, K=0.5$.

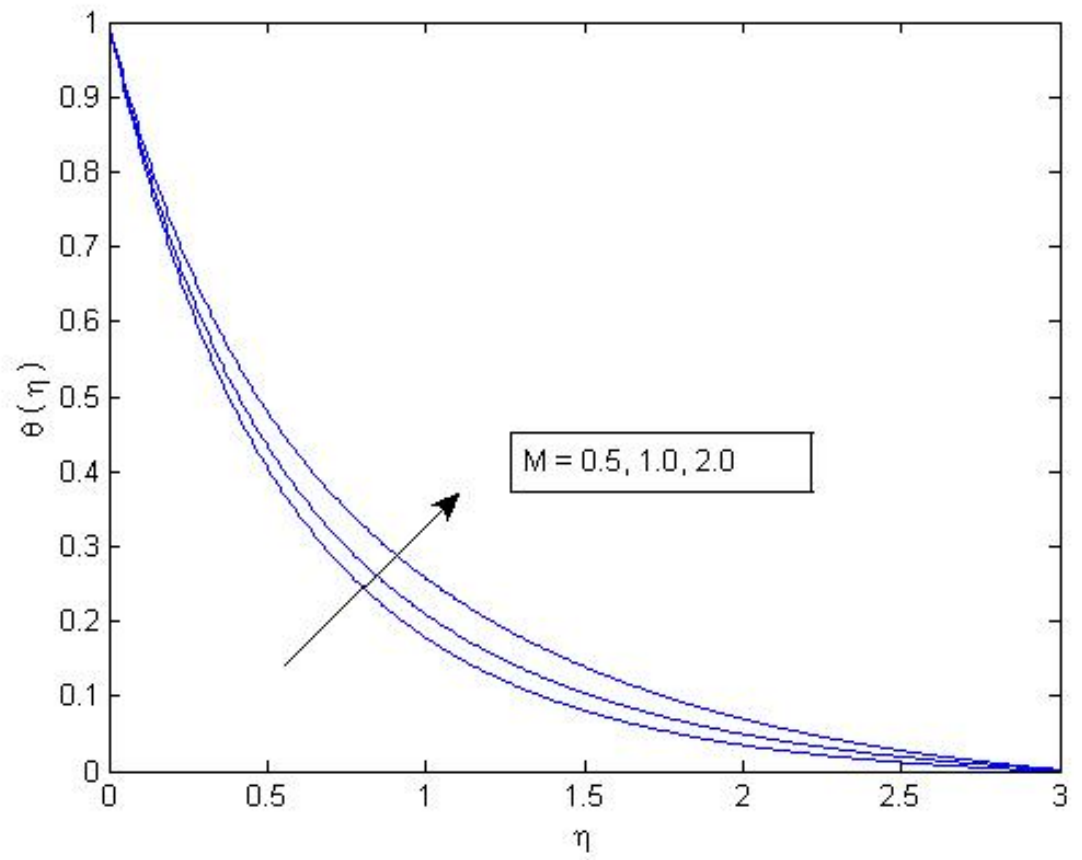

Figure 3 Temperature distribution versus $\eta$ when $\operatorname{Pr}=7, S=0.5, \lambda=0.2, K=0.5, \delta=0.5$. 


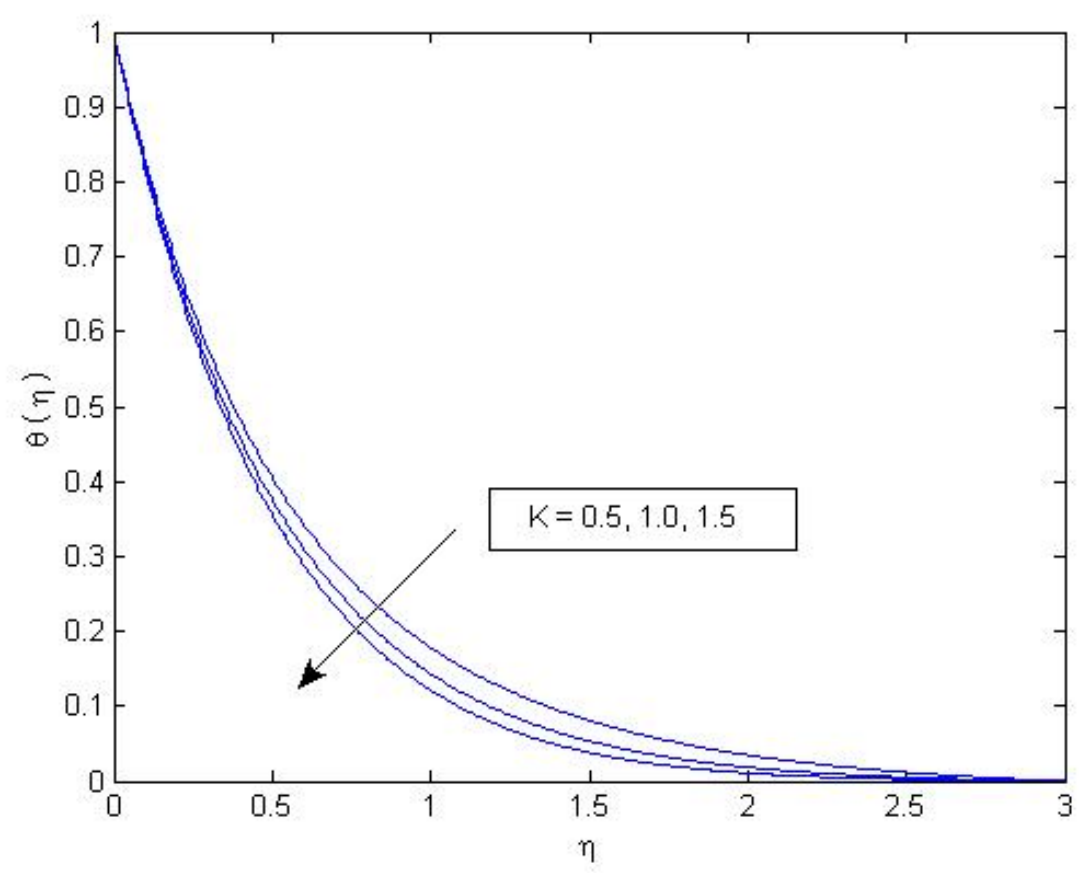

Figure 4 Temperature distribution versus $\eta$ when $\operatorname{Pr}=7, S=0.5, M=0.5, \lambda=0.2, \delta=0.5$.

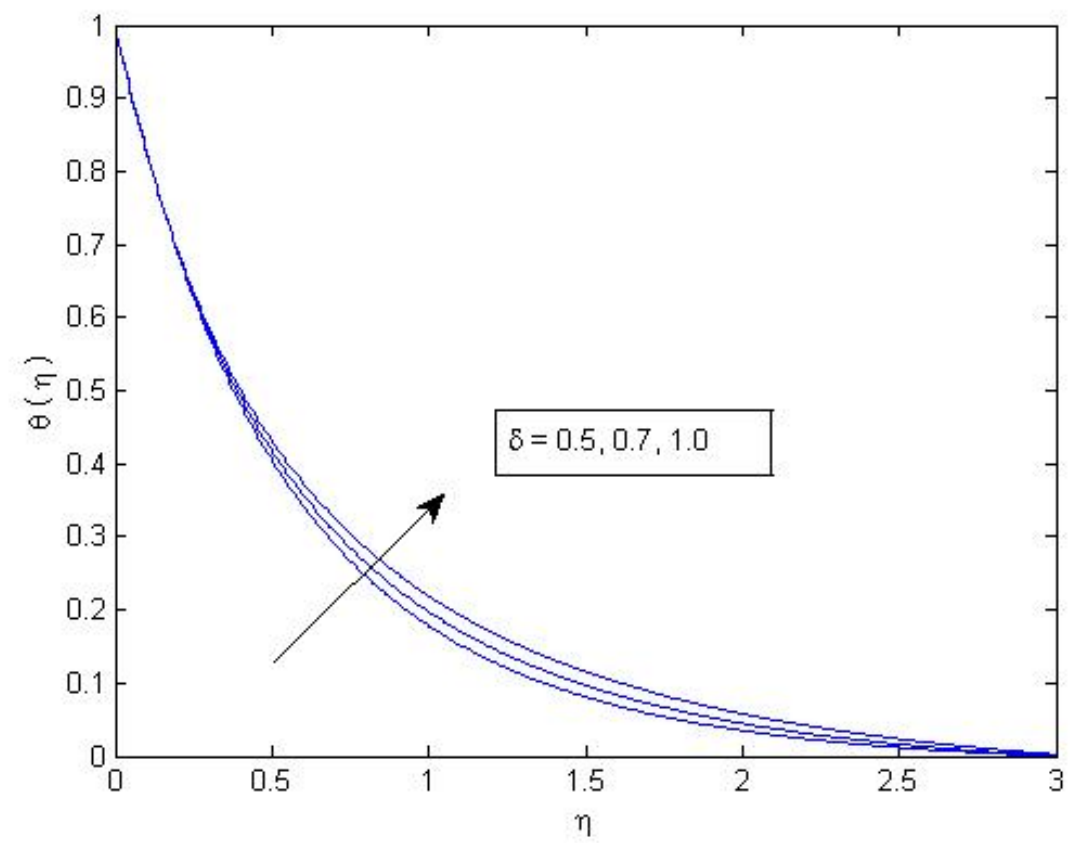

Figure 5 Temperature distribution versus $\eta$ when $P r=7, S=0.5, M=0.5, \lambda=0.2, K=0.5$. 


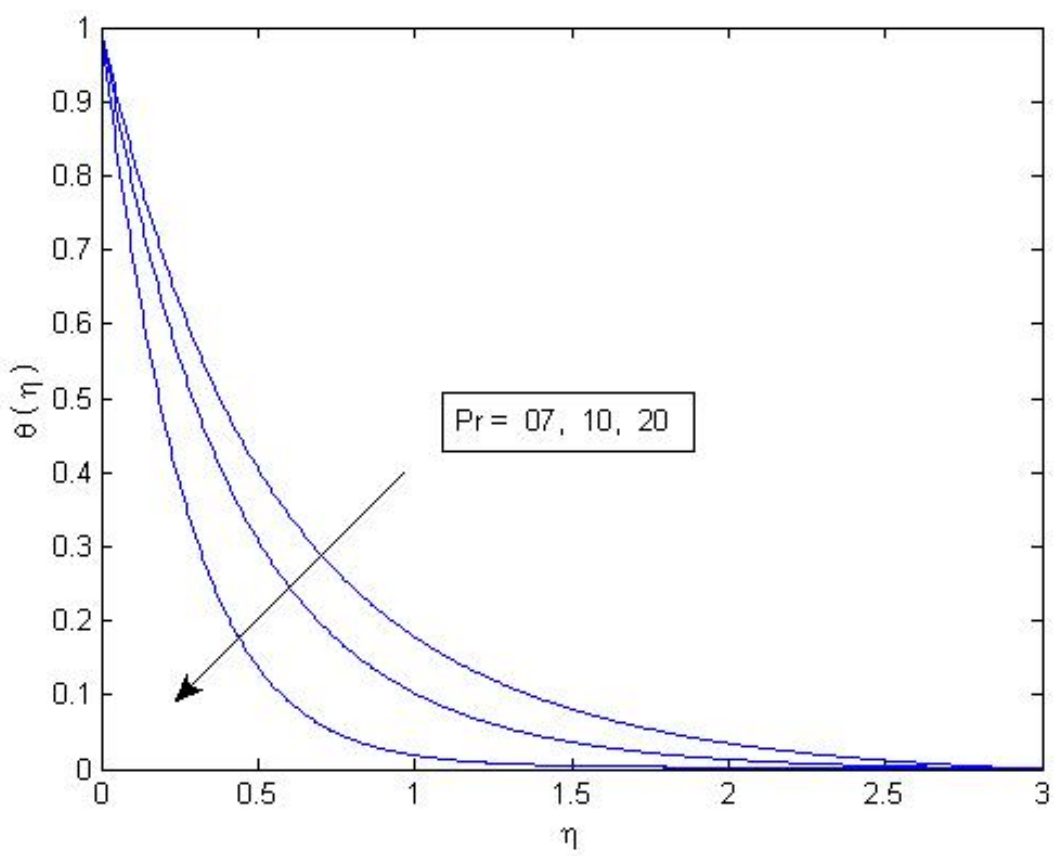

Figure 6 Temperature distribution versus $\eta$ when $S=0.5, M=0.5, \lambda=0.2, K=0.5, \delta=0.5$.

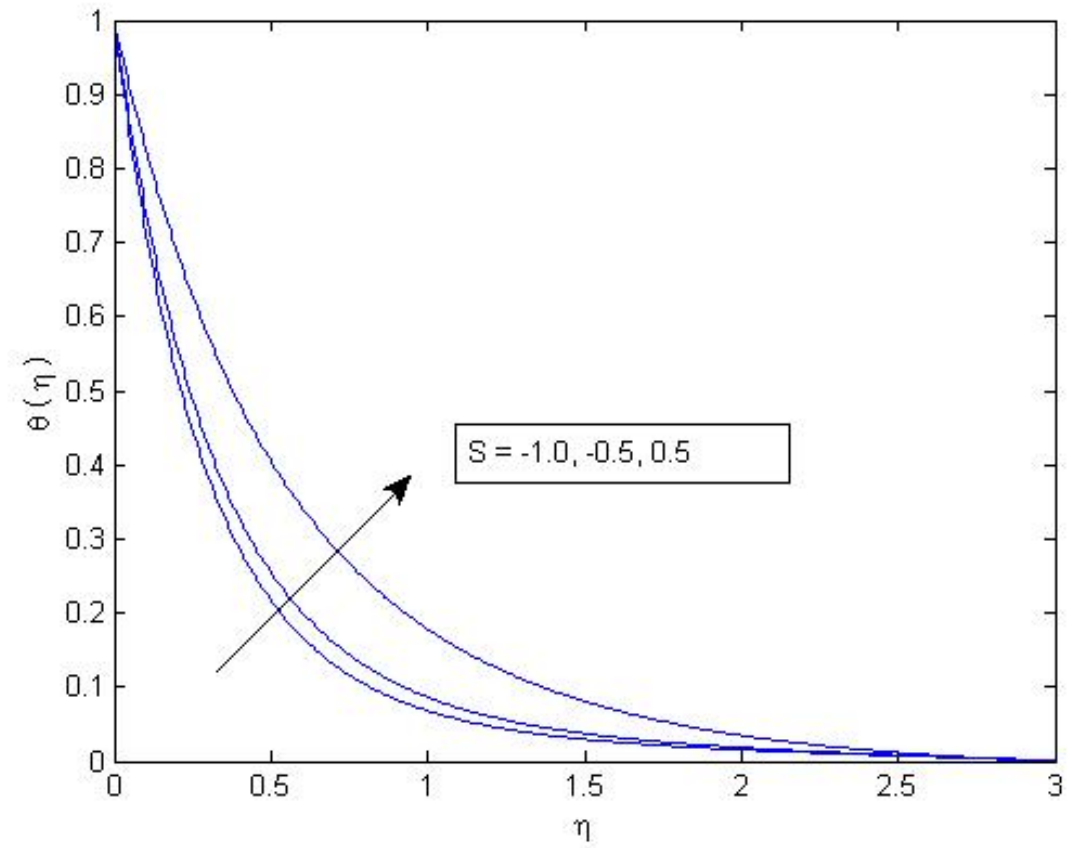

Figure 7 Temperature distribution versus $\eta$ when $\operatorname{Pr}=7, M=0.5, \lambda=0.2, K=0.5, \delta=0.5$. 


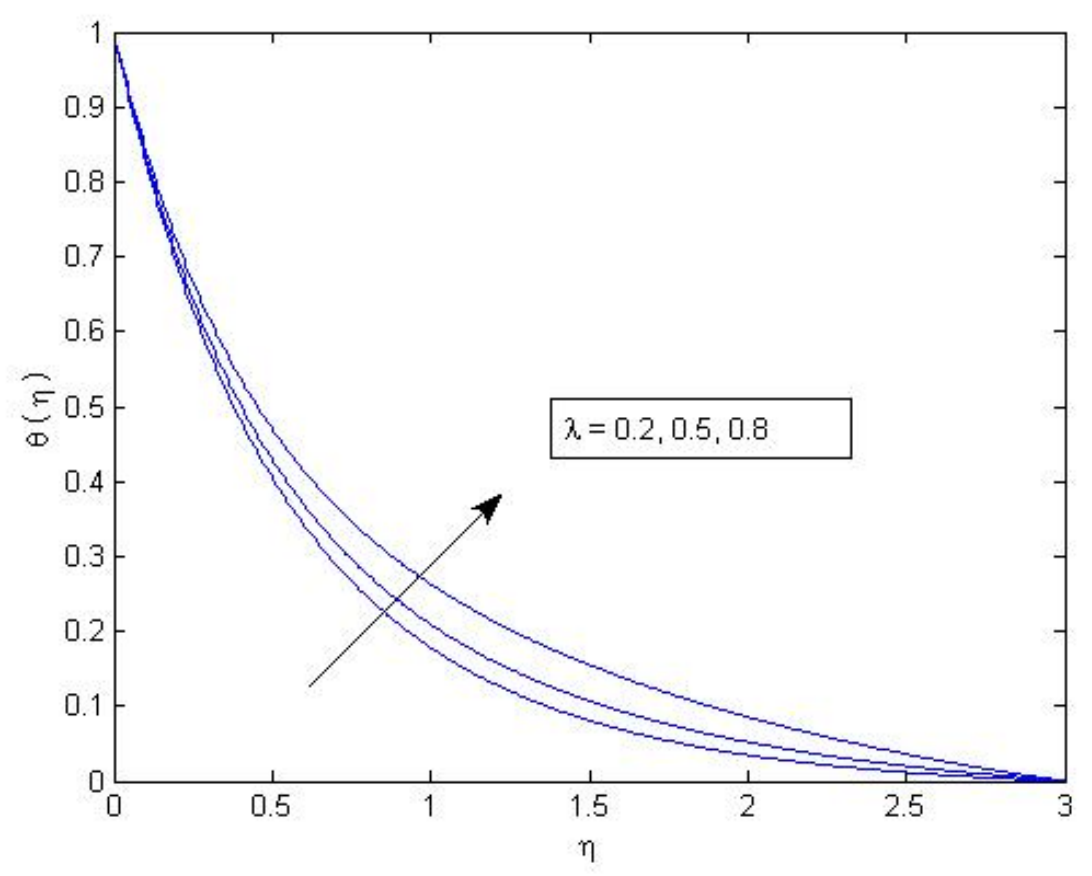

Figure 8 Temperature distribution versus $\eta$ when $P r=7, S=0.5, M=0.5, K=0.5, \delta=0.5$.

Table 1 is prepared to compare the numerical value of Nusselt number in the present study with previously published results by Ishak and Nazar Ishak et al. (2009). The comparison is performed for various values of Prandtl number and it can be noted that the results are in a good agreement with data provided by Ishak et al. (2009). It is observed from Table 2 that shear stress at the surface decreases with increasing Prandtl number, while heat generation/absorption parameter and permeability parameter are having reverse effect on it. The dimensionless rate of heat transfer at the surface increases with increasing Prandtl number, curvature parameter, permeability parameter and it decreases for enhancing heat generation/absorption parameter, magnetic parameter and mixed convection parameter.

Table 2 Numerical values of skin friction coefficient and Nusselt number at the surface for various values of physical parameters.

\begin{tabular}{llllllll}
$\boldsymbol{P r}$ & $\boldsymbol{S}$ & $\boldsymbol{\delta}$ & $\boldsymbol{K}$ & $\boldsymbol{M}$ & $\boldsymbol{\lambda}$ & $\boldsymbol{f}^{\prime \prime}(\mathbf{0})$ & $-\boldsymbol{\theta}^{\prime}(\mathbf{0})$ \\
7 & 0.5 & 0.5 & 0.5 & 0.5 & 0.2 & -1.517001 & 2.290197 \\
10 & 0.5 & 0.5 & 0.5 & 0.5 & 0.2 & -1.524721 & 2.843865 \\
20 & 0.5 & 0.5 & 0.5 & 0.5 & 0.2 & -1.539003 & 4.257002 \\
7 & -1.0 & 0.5 & 0.5 & 0.5 & 0.2 & -2.052012 & 4.065702 \\
7 & -0.5 & 0.5 & 0.5 & 0.5 & 0.2 & -1.839002 & 3.592022 \\
7 & 0.5 & 0.7 & 0.5 & 0.5 & 0.2 & -1.573602 & 2.329505 \\
7 & 0.5 & 1.0 & 0.5 & 0.5 & 0.2 & -1.652602 & 2.397230 \\
7 & 0.5 & 0.5 & 1.0 & 0.5 & 0.2 & -1.517001 & 2.386020 \\
7 & 0.5 & 0.5 & 1.5 & 0.5 & 0.2 & -1.484327 & 2.453002 \\
7 & 0.5 & 0.5 & 0.5 & 1.0 & 0.2 & -1.692702 & 2.181197 \\
\hline
\end{tabular}

Continued on next page 


\begin{tabular}{lllllllll}
\hline Table 2 continued & & & & & & & \\
\cline { 2 - 8 }$y$ & 7 & 0.5 & 0.5 & 0.5 & 2.0 & 0.2 & -1.982703 & 2.009901 \\
7 & 0.5 & 0.5 & 0.5 & 0.5 & 0.5 & -1.517001 & 2.211902 \\
7 & 0.5 & 0.5 & 0.5 & 0.5 & 0.8 & -1.517001 & 2.081971 \\
\hline
\end{tabular}

\section{CONCLUSIONS}

In this article a viscous incompressible Newtonian fluid is considered and its steady flow past a linearly stretching vertical cylinder is investigated. In addition, the flow field is manifested with heat source/sink, external magnetic field and porous medium. The conclusions of the study are itemized as follows:

1. To enhance the fluid velocity, curvature of the cylinder should be taken large

2. Temperature of the fluid can be decreased by increasing permeability parameter or Prandtl number

3. In order to achieve the goal of lesser skin friction, Prandtl number, curvature parameter or magnetic parameter must be increased.

4. For higher rate of heat transfer, larger values of Prandtl number, curvature parameter or permeability parameter can be used.

\section{ACKNOWLEDGEMENTS}

The authors would like to thank the Editor and anonymous reviewers for their careful reading and constructive comments and suggestions, which helped a lot in the improvement of the manuscript.

\section{REFERENCES}

Aydin, O., \& Kaya, A. (2011). MHD-mixed convection from a vertical slender cylinder. Communications in Nonlinear Science and Numerical Simulation, 16(4), 1863-1873. Retrieved from https://dx.doi.org/10.1016/j.cnsns.2010.08.003 10.1016/j.cnsns.2010.08.003

Chang, C. L. (2008). Numerical Simulation For Natural Convection Of Micropolar Fluids Flow Along Slender Hollow Circular Cylinder With Wall Conduction Effect. Communication In Nonlinear Science And Numerical Simulation, 13, 624-636.

Chen, T. S., \& Mucoglu, A. (1975). Bouyancy Effects on Forced Convection Along a Vertical Cylinder. Journal of Heat Transfer, 97(2), 198-203. Retrieved from https://dx.doi.org/ 10.1115/1.3450341 10.1115/1.3450341

Cheng, P. (1977). Similarity Solutions For Mixed Convection From Horizontal Impermeable Surfaces In Saturated Porous Media. International Journal of Heat And Mass Transfer, 20, 893-898.

Crane, L. J. (1970). Flow past a stretching plate. Zeitschrift für angewandte Mathematik und Physik ZAMP, 21(4), 645-647. Retrieved from https://dx.doi.org/10.1007/ bf01587695 10.1007/bf01587695

Ganesan, P. G., , \& Rani, P. H. (2000). Unsteady Free Convection Mhd Flow Past A Vertical Cylinder With Heat And Mass Transfer. Int. J. Therm. Sci, 39, 265-272. 
Grubka, L. J., \& Bobba, K. M. (1985). Heat Transfer Characteristics of a Continuous, Stretching Surface With Variable Temperature. Journal of Heat Transfer, 107(1), 248-250. Retrieved from https://dx.doi.org/10.1115/1.3247387 10.1115/1.3247387

Hayat, T., Anwar, M. S., Farooq, M., \& Alsaedi, A. (2015). Mixed Convection Flow of Viscoelastic Fluid by a Stretching Cylinder with Heat Transfer. PLOS ONE, 10(3), e0118815e0118815. Retrieved from https://dx.doi.org/10.1371/journal.pone.0118815 10 .1371/journal.pone.0118815

Ishak, A., , \& Nazar, R. (2009). Laminar Boundary Layer Flow Along A Stretching Cylinder. European Journal Of Scientific Research, 36, 22-39.

Mishra, U., , \& Singh, G. (2014). Dual Solutions Of Mixed Convection Flow With Momentum And Thermal Slip Flow Over A Permeable Shrinking Cylinder. Computers \& Fluids, 93, 107-115.

Patil, P. M., Roy, S., \& Pop, I. (2012). Unsteady effects on mixed convection boundary layer flow from a permeable slender cylinder due to non-linearly power law stretching. Computers \& Fluids, 56, 17-23. Retrieved from https://dx.doi.org/10.1016/j.compfluid.2011.11 .008 10.1016/j.compfluid.2011.11.008

Reddy, P. B. A., \& Das, R. (2016). Estimation of MHD boundary layer slip flow over a permeable stretching cylinder in the presence of chemical reaction through numerical and artificial neural network modeling. Engineering Science and Technology, an International Journal, 19(3), 1108-1116. Retrieved from https://dx.doi.org/10.1016/j.jestch.2015 .12.013 10.1016/j.jestch.2015.12.013

Sharma, P. R., Sinha, S., Yadav, R. S., \& Filippov, A. N. (2018). MHD mixed convective stagnation point flow along a vertical stretching sheet with heat source/sink. International Journal of Heat and Mass Transfer, 117, 780-786. Retrieved from https://dx.doi.org/10.1016/ j.ijheatmasstransfer.2017.10.026 10.1016/j.ijheatmasstransfer.2017.10.026

Siddheshwar, P. G., , \& Srikrishna, C. V. (2003). Linear And Non-Linear Analysis Of Convection In A Micropolar Fluid Occupying A Porous Medium. Int. J. Non-Linear Mech, 38, 15611579.

Sohut, N. F. H. M., Aziz, A. S. A., \& Ali, Z. M. (Eds.). (2017). Double Stratification Effects On Boundary Layer Over A Stretching Cylinder With Chemical Reaction And Heat Generation. Journal of Physics: Conf. Series, 890, 12019-12020.

Vajravelu, K. (1994). Convection Heat Transfer at a Stretching Sheet with Suction or Blowing. Journal of Mathematical Analysis and Applications, 188(3), 1002-1011. Retrieved from https://dx.doi.org/10.1006/jmaa.1994.1476 10.1006/jmaa.1994.1476

Vinita, V. (2019). And Vikas Poply, Impact Of Outer Velocity Mhd Slip Flow And Heat Transfer Of Nanofluid Past A Stretching Cylinder. Materials Today: Proceedings, 26, 3429-3435.

Wang, C. Y. (1984). The Three-Dimensional Flow Due To A Stretching Flat Surface. Physics of Fluids, 27, 1915-1917.

Wang, C. Y. (2012). Natural Convection On A Vertical Stretching Cylinder. Commun. Nonlinear Sci Numer. Simulat, 17, 1098-1103.

Yadav, R. S., , \& Sharma, P. R. (2014). Effects Of Porous Medium On Mhd Fluid Flow Along A Stretching Cylinder., 6, 104-113. 